\title{
Boundary value problems compatible with symmetries
}

\author{
Burak Gürel, Metin Gürses ${ }^{1}$, Ismagil Habibullin ${ }^{2}$ \\ Department of Mathematics, Faculty of Science, Bilkent University, 06533 Ankara. Turkey
}

Received 1 March 1994; revised manuscript received 4 May 1994; accepted for publication 11 May 1994

Communicated by A.P. Fordy

\begin{abstract}
Boundary value problems for nonlinear differential equations are considered from the point of view of symmetry. In addition to the known ones new families of boundary conditions are found for integrable equations like the Harry-Dym, $\mathrm{KdV}$ and $\mathrm{mKdV}$.
\end{abstract}

It is well known [1-3] that for some classes of completely integrable nonlinear evolution equations,

$$
u_{t}=f\left(u, u_{1}, u_{2}, \ldots, u_{n}\right) \text {, }
$$

where $u=u(x, t), u_{i}=\partial^{i} u / \partial x^{i}$ and $f$ is a scalar (or vector) field, there exist boundary conditions of the form

$$
\left.p\left(u, u_{1}, u_{2}, \ldots, u_{k}\right)\right|_{x=0}=0,
$$

compatible with the inverse scattering transform method or any other attribute of integrability. For instance, Sklyanin [3] has shown that the following boundary value problem on the finite interval $x_{0} \leqslant x \leqslant x_{1}$ for the nonlinear Schrödinger equation $\mathrm{i} u_{t}=u_{x x}+2|u|^{2} u$, with $u_{x}=\left.c_{0} u\right|_{x=x_{0}}$, and $u_{x}=\left.c_{1} u\right|_{x=x_{1}}$, is a completely integrable Hamiltonian system. The main aim of the present paper is to propose a method to obtain boundary conditions for the evolution equations of form (1), which might have applications in the inverse scattering technique. In the proposed method we utilize the generalized symmetries of the nonlinear partial differential equations.

It is worthwhile to remark that all the known boundary conditions of form (2) consistent with the inverse scattering method are indeed compatible with the infinite series of generalized symmetries. On the other hand, stationary solutions of the symmetries compatible with (2) allow one to construct an infinite dimensional set of "exact" (finite gap) solutions of the corresponding boundary value problem (1) and (2). However, in this Letter we do not discuss the analytical aspects of this problem.

\footnotetext{
1 E-mail: gurses@fen.bilkent.edu.tr.

2 Permanent address: Mathematical Institute, Ufa Scientific Center, Russian Academy of Sciences, Chernishevski Street 112 , Ufa 450000, Russian Federation.
} 
In this paper we shall deal with boundary conditions of form (2). An effective investigation of boundary conditions involving an explicit $t$-dependence is essentially more complicated. Such a problem has been studied, for instance, in Ref. [12].

Let the equation

$$
u_{\tau}=g\left(u, u_{1}, \ldots, u_{m}\right) \text {, }
$$

for a fixed value of $m$, be a symmetry of Eq. (1). Let us introduce some new set of dynamical variables consisting of $v=\left(u, u_{1}, u_{2}, \ldots, u_{n-1}\right)$, and its $t$-derivatives $v_{t}, v_{t t}, \ldots$ One can express the higher $x$-derivatives of $u$, i.e., $u_{i}$ for $i \geqslant n$ and their $t$-derivatives, by using Eq. (1) itself, in terms of the dynamical variables $v$ and their $t$-derivatives. Here $n$ is the order of Eq. (1). In these terms symmetry (3) may be written as

$$
v_{\tau}=G\left(v, v_{t}, v_{t t}, \ldots, v_{t t \ldots t}\right)
$$

We call the boundary value problem, Eqs. (1) and (2), compatible with symmetry (3) if the constraint $p(v)=0$ (or the constraints $p^{a}(v)=0$, where $a=1,2, \ldots, N$ and $N$ is the number of constraints) is consistent with the $\tau$-evolution

$$
\frac{\partial p}{\partial \tau}=0 \quad(\bmod p=0)
$$

Eq. (5), by virtue of the equations in (4), must automatically be satisfied. In fact (5) means that the constraint $p=0$ defines an invariant surface in the manifold with local coordinates $\boldsymbol{v}$. This definition of consistency of the boundary value problem with symmetry is closer to the one introduced in Ref. [4], but not identical.

We call the boundary condition (2) compatible with the equation if it is compatible at least with one of its higher order symmetries.

Our main result is that if the boundary condition is compatible with one higher symmetry, then it is compatible with an infinite number of symmetries. In the sequel we suppose that Eq. (1) is integrable, meaning that it admits a recursion operator of the form [5-7]

$$
R=\sum_{i=0}^{i_{1}} \boldsymbol{\alpha}_{i} D^{i}+\sum_{a=0}^{i_{2}} \boldsymbol{\beta}_{a} D^{-1} \gamma_{a}, \quad i_{1}, i_{2} \geqslant 0,
$$

where $\boldsymbol{\alpha}_{i}, \boldsymbol{\beta}_{a}$ and $\gamma_{a}$ are functions of the dynamical variables, $D$ is the total derivative with respect to $x$ and in (6) $i_{2}$ defines the number of nonlocal terms. Recursion operators when applied to a symmetry produce new symmetries. Passing to the new dynamical variables $v$ one can obtain, from (6), the recursion operator of the system of equations (4),

$$
\boldsymbol{R}=\sum_{i=0}^{i_{3}} \boldsymbol{a}_{i}\left(\partial_{t}\right)^{i}+\sum_{a=0}^{i_{4}} \boldsymbol{b}_{a}\left(\partial_{t}^{-1}\right) \boldsymbol{c}_{a}, \quad i_{3}, i_{4}>0,
$$

where $a_{i}, b_{i}$ and $c_{i}$ depend on $v$ and on a finite number of its $t$-derivatives, $\partial_{t}$ is the operator of the total derivative with respect to $t$. In (7) $i_{4}$ is the number of nonlocal terms. If (1) is a scalar equation, $R$ is a scalar operator. Then $\boldsymbol{R}$ is an $n \times n$ matrix valued operator. Our further considerations are based on the following proposition.

Proposition 1. Suppose that the vector field $\boldsymbol{G}$ in (4) may be written as $\boldsymbol{G}=\boldsymbol{R}^{n_{0}} \boldsymbol{v}_{t}$, where $\boldsymbol{R}$ is the recursion operator (7), $n_{0} \geqslant 1$ is an integer. Let the constraint $p(v)=0$ (the rank of the constraint equals $n-1$ ) be consistent with Eq. (4). Then it is consistent with every equation of the form $v_{\tau}=L\left(\boldsymbol{R}^{n_{0}}\right) \boldsymbol{v}_{t}$, where $L=L(z)$ is an arbitrary scalar polynomial of $z$ with constant coefficients. 
As an illustration let us give the Burgers equation as an example. It is given by

$$
u_{t}=u_{x x}+2 u u_{x},
$$

which possesses the recursion operator of the form

$$
R=D+u+u_{x} D^{-1}
$$

(see for instance Ref. [8]).

Proposition 2. If the boundary condition $\left.p\left(u, u_{1}\right)\right|_{x=0}=0$ is compatible with a higher symmetry of the Burgers equation, then it is one of the form $u_{1}+u^{2}+c_{1} u+c_{2}=0$ (see Ref. [4]) or $u=c$ and it is compatible with every symmetry of the form $u_{\tau}=P\left(R^{2}\right) u_{t}$, where $P$ denotes a polynomial with scalar constant coefficients.

Sketch of proof. The Frechet derivative of (8) gives the symmetry equation of the Burgers equation,

$$
\partial_{t} \sigma=\left(D^{2}+2 u D+2 w\right) \sigma,
$$

where $\sigma$ is the symmetry of (8) and $w$ stands for $u_{1}$. As the operators acting on symmetries we may take

$$
D^{-1}=\partial_{t}^{-1}(D+2 u)
$$

in the recursion operator (9). Consequently the recursion formula $u_{\tau_{i+1}}=R u_{\tau_{i}}$ becomes

$$
u_{\tau_{i+1}}=\left(u+2 w \partial_{t}^{-1} u\right) u_{\tau_{i}}+\left(1+w \partial_{t}^{-1}\right) w_{\tau_{i}} .
$$

Differentiating it with respect to $x$ and replacing $w_{x}=u_{2}=u_{t}-2 u w$ one obtains

$$
w_{\tau_{i+1}}=\left[\partial_{t}+2\left(u_{t}-2 u w\right) \partial_{t}^{-1} u\right] u_{\tau_{i}}+\left[-u+\left(u_{t}-2 u w\right) \partial_{t}^{-1}\right] w_{\tau_{i}}
$$

for $i=1,2, \ldots$. Thus the matrix form of the recursion operator $\boldsymbol{R}$ is found as

$$
\boldsymbol{R}=\left(\begin{array}{cc}
u+2 w \partial_{t}^{-1} u & 1+w \partial_{t}^{-1} \\
\partial_{t}+2\left(u_{t}-2 u w\right) \partial_{t}^{-1} u & -u+\left(u_{t}-2 u w\right) \partial_{t}^{-1}
\end{array}\right) .
$$

The important step in our proof is to show that if a boundary condition is compatible with at least one symmetry then it is compatible with the following one,

$$
\left(\begin{array}{l}
u \\
w
\end{array}\right)_{\tau}=\boldsymbol{R}^{2}\left(\begin{array}{l}
u \\
w
\end{array}\right)_{t}
$$

which is exactly the coupled Burgers type integrable system (see Ref. [5], p. 140)

$$
u_{\tau}=u_{t t}+2\left(w+u^{2}\right) u_{t}, \quad w_{\tau}=w_{t t}+2 u_{t}^{2}+2\left(w+u^{2}\right) w_{t} .
$$

It is straightforward to show that the above system (16) is compatible with the constraint $p(u, w)=0$ only if $p=c\left(w+u^{2}\right)+c_{1} u+c_{2}$.

Remark 1. On the invariant surface $p(u, w)=0$ system (16) turns into the Burgers-like equation $u_{\tau}=$ $u_{t t}-2\left(c u+c_{2}\right) u_{t}$, which is also integrable [6]. 
We shall now apply our method to obtain compatible boundary conditions of some other nonlinear partial differential equations. Let us start with the following system of equations,

$$
u_{t}=u_{2}+2 u^{2} v, \quad-v_{t}=v_{2}+2 u v^{2}
$$

Letting $v=u^{*}$ and $t^{*}=-t$ the above system becomes the well known nonlinear Schrödinger equation. Suppose that it admits a boundary condition of the form

$$
\left.u_{x}\right|_{x=0}=p^{1}(u, v),\left.\quad v_{x}\right|_{x=0}=p^{2}(u, v),
$$

compatible with the fourth order symmetry. This means that constraint (18) defines an invariant surface for this symmetry, presented as a system of four equations with two independent variables,

$$
\begin{aligned}
& u_{\tau}=u_{t t}-2 u^{2} v_{t}-4 u v_{1} u_{1}+2 v u_{1}^{2}-2 u^{3} v^{2}, \\
& v_{\tau}=-v_{t}-2 v^{2} u_{t}+4 v u_{1} v_{1}-2 u v_{1}^{2}+2 v^{3} u^{2}, \\
& u_{1, \tau}=u_{1, t t}-2 u^{2} v_{1 t}-2 u_{1}^{2} v_{1}-6 u^{2} v^{2} u_{1}-4 u v_{1} u_{t}+4 v u_{1} u_{t}+4 v u^{3} v_{1}, \\
& v_{1, \tau}=-v_{1, t t}-2 v^{2} u_{1, t}+2 v_{1}^{2} u_{1}+6 v_{1} v^{2} u^{2}-4 v u_{1} v_{t}+4 u v_{1} v_{t}-4 v^{3} u u_{1} .
\end{aligned}
$$

One can check that system (19) is compatible with the constraint $u_{1}=p^{1}(u, v), v_{1}=p^{2}(u, v)$ only if $p^{1}=c u$ and $p^{2}=c v$. Therefore system (19) is of the form

$$
\left(u, u_{1}, v, v_{1}\right)_{\tau}^{\mathrm{T}}=\boldsymbol{R}^{2}\left(u, u_{1}, v, v_{1}\right)_{t}^{\mathrm{T}},
$$

where $\mathrm{T}$ denotes transposition. Hence it follows from Proposition 1 that the constraints $u_{1}=c u, v_{1}=c v$ are compatible with every symmetry of even order. So the boundary conditions $\left.u_{x}\right|_{x=0}=c u,\left.v_{x}\right|_{x=0}=c v$ are compatible with such symmetries. Analytical properties of this boundary value problem were studied previously (see Refs. $[9,10]$ ) by means of the inverse scattering method.

Remark 2. On the invariant surface $u_{1}=c u, v_{1}=c v$ system (19) is reduced to a system of two equations

$$
u_{\tau}=u_{t t}-2 u^{2} v_{t}-2 c^{2} u^{2} v-2 u^{3} v^{2}, \quad v_{\tau}=-v_{t t}-2 v^{2} u_{t}+2 c^{2} u v^{2}+2 u^{2} v^{3} .
$$

Under a suitable change of variables this system of two equations becomes the famous derivative nonlinear Schrödinger equation (see Ref. [5], p. 175).

Among the nonlinear integrable equations the Harry-Dym equation,

$$
u_{t}+u^{3} u_{3}=0
$$

is of special interest. It is not quasilinear, and because of this its analytical properties are not typical. Using the symmetry approach we find a boundary condition of the form

$$
p\left(u, u_{1}, u_{2}\right)=0,
$$

compatible with the Harry-Dym equation. One has to notice that because of the non-quasilinearity of (21) the transformation from the standard set of variables $\left(u, u_{1}, u_{2}, u_{3}, \ldots\right)$ to $\left(u, u_{1}, u_{2}, u_{t}, u_{1, t}, u_{2, t}, \ldots\right)$ is not regular. For instance $u_{3}=-u_{t} / u^{3}$. It has a singular surface given by the equation $u=0$. So one should examine this surface separately. Since the Harry-Dym equation (21) as well as its higher order symmetries possess the reflection symmetry $x \rightarrow-x, u \rightarrow-u, t \rightarrow t$ the trivial boundary condition $u(t, 0)=0$ is consistent with integrability. 
Suppose that the boundary value problem (21) and (22) is compatible with the ninth order symmetry $u_{\tau}=u^{9} u_{9}+\ldots$. This means that the constraint $p(u, v, w)$ is consistent with the following system of equations, equivalent to the ninth symmetry,

$$
u_{\tau}=f_{1}, \quad v_{\tau}=f_{2}, \quad w_{\tau}=f_{3},
$$

where $v=u_{x}, w=u_{x x}$ and $\left(f_{1}, f_{2}, f_{3}\right)^{\mathrm{T}}=\boldsymbol{R}^{3}\left(u_{t}, v_{t}, w_{t}\right)^{\mathrm{T}}$. Here $\boldsymbol{R}$ is given by

$$
\boldsymbol{R}=\left(\begin{array}{ccc}
u w+u_{t} \partial_{t}^{-1} w & -u v-u_{t} \partial_{t}^{-1} v & u^{2}+u_{t} \partial_{t}^{-1} u \\
(1 / u) \partial_{t}+v w-u_{t} / u^{2}+v_{t} \partial_{t}^{-1} w & -v^{2}-v_{t} \partial_{t}^{-1} v & u v+v_{t} \partial_{t}^{-1} u \\
w^{2}+w_{t} \partial_{t}^{-1} w & (1 / u) \partial_{t}-v w-u_{t} / u^{2}-w_{t} \partial_{t}^{-1} v & u w+w_{t} \partial_{t}^{-1} u
\end{array}\right) .
$$

The explicit expressions for $f_{2}$ and $f_{3}$ are very long. Here we give only the function $f_{1}$,

$$
f_{1}=-u_{t t t}+3 u_{t t} u_{t} / u-\frac{3}{2} u_{t t} u_{1} h-\frac{3}{2} u_{t}^{3} / u^{2}+\frac{3}{2} u u_{1, t} h+\frac{3}{2} u u_{1, t} h_{t}-\frac{15}{16} u h^{2} h_{t}-\frac{5}{16} h^{3} u_{t}-\frac{3}{2} u_{1} u_{t} h_{t},
$$

where $h=2 u_{2} u-u_{1}^{2}$. Here one has two choices for the rank of Eq. (22). It is either one or two. The first choice does not lead to any regular invariant surface. The second gives

$$
\left.u_{x}\right|_{x=0}=c u,\left.\quad u_{x x}\right|_{x=0}=\frac{1}{2} c^{2} u .
$$

Remark 3. On the invariant surface $v=c u, w=\frac{1}{2} c^{2} u$ system (23) takes the form

$$
u_{\tau}=-u_{t t t}+\frac{3 u_{t} u_{t t}}{u}-\frac{3 u_{t}^{3}}{2 u^{2}}
$$

which is equivalent to the MKdV equation.

Since the symmetry under consideration is of the form $u_{\tau}=R^{3} u_{x}$ where $R=u^{3} D^{3} u D^{-1}\left(1 / u^{2}\right)$ is the recursion operator for the Harry-Dym equation (see Ref. [11]), Propositon 1 implies the following proposition.

Proposition 3. The boundary value problem (21) and (22) is compatible with every symmetry of the form $u_{\tau}=L\left(R^{3}\right) u_{x}$, where $L$ is a polynomial with scalar constant coefficients.

The Korteweg-de Vries equation $u_{t}=u_{x x x}+6 u_{1} u$ admits a recursion operator $R=D^{2}+4 u+2 u_{1} D^{-1}$ which may be represented in the form

$$
\boldsymbol{R}=\left(\begin{array}{ccc}
4 u+12 v \partial_{t}^{-1} u & 0 & 1+2 v \partial_{t}^{-1} \\
\partial_{t}+12 w \partial_{t}^{-1} u & -2 u & 2 w \partial_{t}^{-1} \\
2 w+12\left(u_{t}-6 u v\right) \partial_{t}^{-1} u & \partial_{t}-2 v & -2 u+2\left(u_{t}-6 u v\right) \partial_{t}^{-1}
\end{array}\right)
$$

It is not difficult to show that the system of equations $(u, v, w)_{\tau}=\boldsymbol{R}^{3}(u, v, w)_{t}$ admits an invariant surface $u=0, w=0$ on which the system turns into the $\mathrm{mKdV}$ equation. This means that the boundary conditions $u(t, x=0)=0, u_{x x}(t, x=0)=0$ are compatible with all symmetries of the form $u_{\tau}=R^{3 n} u_{x}$. Similarly, the $\mathrm{mKdV}$ equation $u_{t}=u_{x x x}+6 u^{2} u_{x}$ is compatible with the boundary condition $u(t, x=0)=0, u_{x}(t, x=0)=0$.

It is easy to see that any symmetry of Eq. (1) rewritten in terms of the nonstandard set of the dynamical variables turns into the equation containing $n-1$ extra variables $u_{1}, u_{2}, \ldots, u_{n-1}$. For instance, the fourth order symmetry of the Burgers equation,

$$
u_{\tau}=u_{4}+4 u_{3} u+10 u_{2} u_{1}+6 u_{2} u^{2}+12 u_{1}^{2} u+4 u_{1} u^{3},
$$

takes the following form,

$$
u_{\tau}=u_{t t}+2\left(u_{1}+u^{2}\right) u_{t},
$$


where $w=u_{1}$.

In the method proposed so far the aim was to find a compatible constraint $p(u, w)=0$ for the system $(u, w=$ $u_{1}$ ) given in (16). There is however a shorter algorithm to examine the existence of compatible boundary conditions. This way we find a much larger class of boundary conditions than in the previous case. We call such boundary conditions weakly compatible with the symmetries. In general $(27)$ is a $(2+1)$-dimensional equation. The next step in our algorithm is to reduce $(27)$ to a $(1+1)$-dimensional equation. This is done by writing (27) on the surface $x=0$ in $(\tau, t, x)$ space. If $u=q$ and $u_{1}=s$ on this surface, then we have

$$
q_{\tau}=q_{t t}+2\left(s+q^{2}\right) q_{t} .
$$

The last step is to require (28) to be integrable. In the general case this is the most difficult part of the algorithm. To find an integrable subclass of equations there are several equivalent methods. For the above equation the whole classification is known [5]. The function $s$ must be of the form $s=-q^{2}+c_{1} q++c_{2}$, where $c_{1}$ and $c_{2}$ are arbitrary constants. This is exactly the same result as we obtained earlier.

As stated in our remarks above, the compatibility of a boundary condition with a symmetry implies weak compatibility, but not vice versa. As an example we give the Harry-Dym equation. Let us consider its fifth order symmetry,

$$
u_{\tau_{5}}=-\frac{1}{2} u^{3}\left(2 u_{5} u^{2}+10 u_{4} u_{1} u+10 u_{3} u_{2} u+5 u_{3} u_{1}^{2}\right),
$$

which may be written in the form $u_{\tau_{5}}=h_{t}$, where $h=2 u_{2} u-u_{1}^{2}$. We also give the next two symmetries in the similar form,

$$
u_{\tau_{7}}=u_{t t} u_{1}-\frac{3}{2} u_{t} u_{1} u h+\frac{3}{8} u_{t}\left[3\left(h+u_{1}^{2}\right)^{2}-4 u_{1}^{2}\left(h+u_{1}^{2}\right)+u_{1}^{4}\right]-u u_{1 t t}+\frac{3}{8} u_{2 t} u h
$$

and $u_{\tau_{9}}=f_{1}$ (where $f_{1}$ is given in (24)). It is evident that for an arbitrary function $F=F(u)$ the constraint $h=0, u_{1}=F(u)$ is weakly consistent with the fifth and ninth symmetries, because the former takes the trivial form $u_{\tau_{5}}=0$ and the latter turns into the integrable equation (26). The seventh order symmetry becomes $u_{\tau_{7}}=\left(S u_{t}\right)_{t}$, where $S=F-u F^{\prime}$. Thus, if $S=1 /(\gamma u+\beta)^{2}$, then the equation $u_{\tau_{7}}=\left(S u_{t}\right)_{t}$ will have to be integrable (see Ref. [6]). Supposing $S(u)=a$ one can easily find that $u_{1}=c u+a, u_{2}=\frac{1}{2} c^{2} u+a c+a^{2} / 2 u$. This leads to the following boundary condition, $u_{x}=c u+a, u_{x x}=u_{x}^{2} / 2 u$, at $x=0$ for the Harry-Dym equation, which coincides with our previous result (25) if $a=0$. To find $F$ in the case $S=1 /(\gamma u+\beta)^{2}$ one has to integrate the ordinary differential equation $F(u)-u F^{\prime}(u)=S$.

We thank G. Alekseev and E. Ferapontov for useful discussions. This work has been partially supported by the Turkish Scientific and Technical Research Council (TUBITAK). One of us (I.H.) thanks TUBITAK and the Russian Foundation for Fundamental Research, grant 93-011-165 for partial support and Bilkent University for the warm hospitality.

\section{References}

[1] M. Kac and P. van Moerbeke, Adv. Math. Ser. 49 (1975) 23.

[2] M. Ablowitz and H. Segur, J. Math. Phys. 16 (1975) 1054.

[3] E.K. Sklyanin, Func. Anal. Prilozh. 21 (1987) 86.

[4] I.T. Habibullin, Phys. Lett. A 178 (1993) 369.

[5] A.V. Mikhailov, V.V. Sokolov and A.B. Shabat, in: What is integrability? (Springer, Berlin, 1991).

[6] A.S. Fokas, J. Math. Phys. 21 (1980) 1318; SIAM 77 (1987) 253.

[7] M. Gürses, A. Karasu and A. Satir, in: Nonlinear evolution equations and dynamical systems, NEEDS'91, eds. M. Boiti, L. Martinelli and F. Pempinelli (World Scientific, Singapore, 1992).

[8] P. Olver, Applications of Lie groups to differential equations (Springer, Berlin, 1986). 
[9] P.N. Bibikov and V.O. Tarasov, Teor. Mat. Fiz. 79 (1989) 334.

[10] I.T. Khabibullin, Teor. Mat. Fiz. 86 (1991) 130.

[11] M. Leo, R.A. Leo, G. Soliani and L. Solombrino, Lett. Nuovo Cimento 38 (1983) 45.

[12] A.S. Fokas and A.R. Its, Phys. Rev. Lett. 68 (1992) 3117. 